\title{
Metallomics
}

Cite this: Metallomics, 2013, 5, 1031

Received 1st February 2013, Accepted 21st May 2013

DOI: $10.1039 / c 3 m t 00039 g$

www.rsc.org/metallomics

\section{In vitro intestinal bioavailability of arsenosugar metabolites and presystemic metabolism of thio-dimethylarsinic acid in Caco-2 cells}

\author{
Larissa Leffers, $\uparrow^{\mathrm{ab}}$ Christoph A. Wehe, $\dagger^{\mathrm{c}}$ Sabine Hüwel, ${ }^{\mathrm{d}}$ Marc Bartel, ${ }^{\mathrm{b}}$ \\ Franziska Ebert, ${ }^{\mathrm{b}}$ Mojtaba S. Taleshi, ${ }^{\mathrm{e}}$ Hans-Joachim Galla, ${ }^{\mathrm{d}}$ Uwe Karst, $^{\mathrm{c}}$ \\ Kevin A. Francesconi ${ }^{\mathrm{e}}$ and Tanja Schwerdtle ${ }^{\star a b}$
}

\begin{abstract}
Whereas inorganic arsenic is classified as a human carcinogen, risks to human health related to the presence of arsenosugars in marine food are still unclear. Since studies indicate that human inorganic arsenic metabolites contribute to inorganic arsenic induced carcinogenicity, a risk assessment for arsenosugars should also include a toxicological characterization of their respective metabolites. Here we assessed intestinal bioavailability of the human arsenosugar metabolites oxo-DMAA ${ }^{\vee}$, thio-DMAA ${ }^{\mathrm{V}}$, oxo-DMAE ${ }^{\mathrm{V}}$, thio-DMAE ${ }^{\mathrm{V}}$ and thio-DMA ${ }^{\vee}$ in relation to arsenite in the Caco-2 intestinal barrier model. Whereas arsenite and thio-DMA ${ }^{\vee}$ caused barrier disruption at concentrations $\geq 10 \mu \mathrm{M}$, all other metabolites did not cause a barrier leakage, even when applied at 50 times higher concentrations than arsenite and thio-DMA ${ }^{\mathrm{V}}$. The transfer studies point to a strong intestinal bioavailability of thio-DMA ${ }^{\vee}$ and thio-DMAE ${ }^{\vee}$, whereas oxo-DMAA ${ }^{\vee}$, thio-DMAA ${ }^{\vee}$ and oxo-DMAE ${ }^{\vee}$ passed the in vitro intestinal barrier only to a very small extent. Detailed influx and efflux studies indicate that arsenite and thio-DMA ${ }^{\vee}$ cross the intestinal barrier most likely by passive diffusion (paracellular) and facilitated (transcellular) transport. LC-ICP-QMS based arsenic speciation studies during the transfer experiments demonstrate transfer of thio-DMA ${ }^{\vee}$ itself across the intestinal barrier and suggest metabolism of thio-DMA ${ }^{\mathrm{V}}$ using the in vitro intestinal barrier model to its oxygen-analogue $\mathrm{DMA}^{\mathrm{V}}$. In the case of arsenite no metabolism was observed. In summary the two arsenosugar metabolites thio-DMA ${ }^{\vee}$ and thio-DMAE ${ }^{\vee}$ showed intestinal bioavailability similar to that of arsenite, and about 10-fold higher than that reported for arsenosugars (Leffers et al., Mol. Nutr. Food Res., 2013, DOI: 10.1002/mnfr.201200821) in the same in vitro model. Thus, a presystemic metabolism of arsenosugars might strongly impact arsenic intestinal bioavailability after arsenosugar intake and should therefore be considered when assessing the risks to human health related to the consumption of arsenosugar-containing food.
\end{abstract}

\section{Introduction}

Due to the ubiquitous environmental distribution of arsenic in water, air, soil and food, humans are frequently exposed to

\footnotetext{
${ }^{a}$ Graduate School of Chemistry, University of Münster, Wilhelm-Klemm-Straße 10, 48149 Münster, Germany

${ }^{b}$ Institute of Food Chemistry, University of Münster, Corrensstraße 45, 48149 Münster, Germany. E-mail: Tanja.Schwerdtle@uni-muenster.de; Fax: +49-251-83-33396; Tel: +49-251-83-33874

${ }^{c}$ Institute of Chemistry - Analytical Chemistry, University of Münster, Corrensstraße 28/30, 48149 Münster, Germany

${ }^{d}$ Institute of Biochemistry, University of Münster, Wilhelm-Klemm-Straße 2, 48149 Münster, Germany

${ }^{e}$ Institute of Chemistry - Analytical Chemistry, University of Graz, Universitaetsplatz 1, Graz, Austria

$\dagger$ Both authors contributed equally to the practical work of this study.
}

inorganic and organic arsenic species. ${ }^{1}$ Inorganic arsenic is classified as a human carcinogen, both via ingestion and inhalation routes. ${ }^{2,3}$ Health risks associated with the naturally occurring organic arsenicals are still unclear. ${ }^{1}$ Organic arsenic species, including arsenobetaine, arsenosugars and arsenolipids, are naturally present in high concentrations in marine products. ${ }^{4}$ Additionally, a variety of organic arsenic species, including monoand dimethylated trivalent and pentavalent arsenicals, are formed during inorganic arsenic metabolism. ${ }^{5-8}$ In vivo data, both in experimental animals and humans, and in vitro data indicate that some of these metabolites strongly contribute to inorganic arsenic induced toxicity. ${ }^{1,9,10}$ Thus, in cultured human cells the trivalent methylated metabolites monomethylarsonous acid $\left(\mathrm{MMA}^{\mathrm{III}}\right)$ and dimethylarsinous acid (DMA $\left.{ }^{\mathrm{III}}\right)$ generally induce stronger toxic effects as compared to arsenite (e.g. ref. 11-15). 
Briefly summarized, during the last few years the assumption has arisen that human inorganic arsenic metabolism plays a key role in inorganic arsenic induced carcinogenicity.

Hence, research has focused on arsenic speciation in biological samples to better understand not only inorganic arsenic metabolism but also arsenosugar metabolism in mammals, and to identify new arsenic metabolites (e.g. ref. 5, 16 and 17). Thio-dimethylarsinic acid (thio-DMA ${ }^{\mathrm{V}}$ ) has been identified as a new human inorganic arsenic metabolite. ${ }^{18-20}$ In contrast to arsenobetaine, which is not metabolized in humans, arsenosugars are metabolised and lead to a broad spectrum of several toxicologically uncharacterized arsenic species. Thus, after the ingestion of arsenosugars, the metabolites oxo- and thiodimethylarsenoacetate (oxo-DMAA ${ }^{\mathrm{V}} /$ thio-DMAA $^{\mathrm{V}}$ ), oxo- and thio-dimethylarsenoethanol (oxo-DMAE ${ }^{\mathrm{V}}$ /thio-DMAE ${ }^{\mathrm{V}}$ ), dimethylarsinic acid $\left(\mathrm{DMA}^{\mathrm{V}}\right)$ and thio-DMA ${ }^{\mathrm{V}}$ were detected in human urine. $^{5,21,22}$

Some of these arsenic metabolites might also directly be present in food, which has previously been shown for thio$\mathrm{DMA}^{\mathrm{V}}$ in rice. ${ }^{23}$ Additionally, these metabolites might occur in the gastrointestinal tract (GI tract) due to biotransformation of arsenosugars by the GI tract microbiota and due to enterohepatic circulation. ${ }^{24-28}$ In this context, very recently it has been demonstrated that two food-relevant arsenosugars exhibit only low intestinal bioavailability applying the Caco- 2 model. ${ }^{29}$ Thus a presystemic metabolism of the arsenosugars should be considered.

Differentiated Caco-2 cells are a well-established, frequently used and well-accepted in vitro model to mimic the intestinal barrier. ${ }^{30-32}$ Previous studies, using this in vitro model, have predicted good absorption properties for arsenite with absorptive permeability coefficients between 1.1-4.6 $\times 10^{-6} \mathrm{~cm} \mathrm{~s}^{-1} \cdot{ }^{33-35}$ In comparison to arsenite, $\mathrm{DMA}^{\mathrm{III}}$ and $\mathrm{MMA}^{\mathrm{III}}$ show stronger intestinal bioavailability (permeability coefficients between $\left.8-12 \times 10^{-6} \mathrm{~cm} \mathrm{~s}^{-1}\right) .{ }^{34}$ Permeability coefficients for the corresponding pentavalent arsenic species are in general 10-fold lower. The studies indicate moderate absorption for arsenate $\left(\mathrm{iAs}^{\mathrm{V}}\right)$, whereas $\mathrm{DMA}^{\mathrm{V}}$ and monomethylarsonic acid $\left(\mathrm{MMA}^{\mathrm{V}}\right)$ show low absorption. ${ }^{34,36}$ For thio-DMA ${ }^{\mathrm{V}}$, oxo-DMAE ${ }^{\mathrm{V}}$, thio-DMAE ${ }^{\mathrm{V}}$, Oxo-DMAA $^{\mathrm{V}}$ and thio-DMAA ${ }^{\mathrm{V}}$, no intestinal bioavailability studies have been published so far.

In this study, differentiated Caco- 2 cells were used as an in vitro model to assess for the first time the impact of thio$\mathrm{DMA}^{\mathrm{V}}$, oxo-DMAE ${ }^{\mathrm{V}}$, thio-DMAE ${ }^{\mathrm{V}}$, oxo-DMAA ${ }^{\mathrm{V}}$ and thio-DMAA ${ }^{\mathrm{V}}$ on the intestinal barrier and to investigate their intestinal bioavailability in relation to arsenite (Fig. 1). The differentiated Caco-2 cells were grown on Transwell ${ }^{\circledR}$ filters to build a two chamber model, with the cell layer resembling the intestinal barrier, the upper apical side referring to the intestinal lumen and the lower basolateral side referring to the blood side. The studies focused on long term exposure (48 h), thereby mimicking a continued exposure towards the arsenic species. The transfer of the arsenicals from the apical to the basolateral side, and in the case of thio-DMA ${ }^{\mathrm{V}}$ and arsenite, transfer in both directions, cellular retention and intestinal metabolism were measured.

\begin{tabular}{|c|c|c|}
\hline Structure & Name & Abbreviation \\
\hline & oxo-dimethylarsenoacetic acid & oxo-DMAAV \\
\hline & thio-dimethylarsenoacetic acid & thio-DMAA ${ }^{\mathrm{V}}$ \\
\hline & oxo-dimethylarsenoethanol & oxo-DMAEV \\
\hline & thio-dimethylarsenoethanol & thio-DMAEV \\
\hline & dimethylarsinic acid & $\mathrm{DMA}^{\mathrm{V}}$ \\
\hline & thio-dimethylarsinic acid & thio-DMAV \\
\hline 1 & arsenite (arsenious acid) & iAs \\
\hline
\end{tabular}

Fig. 1 Chemical structures, names, and abbreviations of the seven arsenic species investigated in this study.

\section{Materials and methods}

\section{Caution}

Inorganic arsenic is classified as a human carcinogen. The following chemicals are hazardous or potentially hazardous and should be handled with care: sodium (meta)-arsenite, oxo-DMAA ${ }^{\mathrm{V}}$, oxo-DMAE ${ }^{\mathrm{V}}$, thio-DMAA ${ }^{\mathrm{V}}$, thio-DMAE ${ }^{\mathrm{V}}, \mathrm{DMA}^{\mathrm{V}}$ and thio-DMA ${ }^{\mathrm{V}}$.

\section{Materials}

Trypsin, foetal calf serum (FCS), penicillin-streptomycin solutions and bovine serum albumin (BSA) were from PAA (Cölbe, Germany). Sodium (meta)-arsenite ( $\geq 99 \%$ purity) was from Fluka Biochemika (Buchs, Germany); neutral red dye, oxo-DMA ${ }^{\mathrm{V}}$ ( $\geq 99 \%$ purity), PMSF, sodium desoxycholate, aprotinin, leupeptin, pepstatin, sodium dodecylsulfate and Hoechst 33258 (bisbenzimide) were supplied by Sigma-Aldrich (Steinheim, Germany). Mouse antioccludin, alexa fluor ${ }^{\circledR} 546$ goat anti-mouse and Aqua Poly/Mount were from Zytomed (Berlin, Germany), Invitrogen (Paisley, UK) and Polysciences (Washington, USA), respectively. Eagle's minimum essential medium (MEM), non-essential amino acids and the culture dishes were obtained from Biochrom (Berlin, Germany). Hydrogen peroxide solution $\left(30 \%\right.$, Suprapur $\left.{ }^{\circledR}\right)$ and nitric acid (65\%, Suprapur) were products of Merck (Darmstadt, Germany) and Bradford solution was from Bio-Rad (Munich, Germany). Cell counting kit- ${ }^{\mathbb{R}}$ was obtained from Dojindo Molecular Technologies (Munich, Germany). The ICP-MS elemental standard (As, $1 \mathrm{mg} \mathrm{mL}{ }^{-1}$ ) was purchased from Spectec (Erding, Germany). All other pro-analysis chemicals were obtained from Sigma-Aldrich (Steinheim, Germany) or Merck (Darmstadt, Germany).

Synthesis, analysis and purity control of the arsenic species and preparation of respective stock solutions

Thio-DMA ${ }^{\mathrm{V}}$, oxo- and thio-DMAA ${ }^{\mathrm{V}}$, oxo- and thio-DMAE ${ }^{\mathrm{V}}$ were synthesised and purified as published before., ${ }^{5,37-39}$ 
The compounds were characterized and their purity was established using ${ }^{1} \mathrm{H}$ and ${ }^{13} \mathrm{C}$ NMR spectroscopy, molecular mass spectrometry (electrospray ionization; single quadrupole mass spectrometer), and LC-elemental mass spectrometry (ICP-MS). When purity was verified, each of the compounds was treated as follows. A portion (10-20 mg) of the arsenical was dissolved in $10 \mathrm{~mL}$ water (Milli-Q, a resistivity of $18.2 \mathrm{MU} \mathrm{cm}$, Millipore $\mathrm{GmbH}$ Vienna, Austria), and the solution was checked for arsenic concentration using ICP-MS and for "arsenic species purity" using LC-ICP-MS, which was $>99.5 \%$ in all cases. Occasionally traces of $\mathrm{DMA}^{\mathrm{V}}$, a byproduct from the reaction, were detected; inorganic arsenic was not detectable.

Arsenic species stock solutions were prepared in sterile bi-distilled water (10-100 mM). All stock solutions were prepared shortly before each experiment to prevent degradation.

\section{Cell culture}

The Caco-2 cells were obtained from the European Collection of Cell Cultures (ECACC; number 86010202, Salisbury, U.K.). Caco-2 cells were grown as a monolayer in culture dishes in MEM supplemented with FCS $(10 \%, \mathrm{v} / \mathrm{v})$, non-essential amino acids $(1 \%, \mathrm{v} / \mathrm{v})$, glutamine (2 mM), penicillin (100 $\left.\mathrm{U} \mathrm{mL}^{-1}\right)$ and streptomycin $\left(100 \mu \mathrm{g} \mathrm{mL}^{-1}\right)$. The cultures were incubated at $37{ }^{\circ} \mathrm{C}$ with $5 \% \mathrm{CO}_{2}$ in air and $100 \%$ humidity. Subconfluent cultures (70-80\%) were split every 2 to 3 days in a $1: 3$ ratio using $0.25 \%$ trypsin solution. Before transfer experiments, cells were thawed and sub-cultured three times to achieve a stable phenotype. For transfer experiments, cells were seeded at a density of $5 \times 10^{4}$ cells per $\mathrm{cm}^{2}$ and were cultured for 11 days until complete differentiation. During this time, the culture medium was replaced every 2-3 days. All experiments were carried out with cells in passage number 53 on day 11 after initial seeding.

\section{Cytotoxicity testing}

Cytotoxicity of the arsenic species in Caco-2 cells was evaluated using the neutral red uptake assay and the CCK- $8^{\circledR}$ assay, assessing the impact of the arsenicals on lysosomal integrity and cellular dehydrogenase activity, respectively. All culture conditions, including media and cell density, were conducted as described above. Both tests were carried out as published before. ${ }^{40}$ Briefly, in the case of the neutral red uptake assay the cells were cultured in 96-well culture plates and incubation with the respective arsenic species was performed on day 11. After 24 and $48 \mathrm{~h}$ incubation, the medium was replaced by neutral red (3-amino-7-dimethylamino-2-methylphenazine hydrochloride) containing medium (70 $\mathrm{mg} \mathrm{L}^{-1}$ neutral red). After dye loading $\left(37^{\circ} \mathrm{C}, 3 \mathrm{~h}\right)$, cells were washed with phosphate-buffered saline (PBS) containing $0.5 \%$ formaldehyde, the incorporated dye was solubilised in $100 \mu \mathrm{L}$ acidified ethanol solution (50\% ethanol, 1\% acetic acid in PBS) and finally the absorbance in each well was measured using a plate reader (Invinite 200Pro, Tecan Group Ltd., Männedorf, Switzerland) at $540 \mathrm{~nm}$.

To measure the effects on cellular dehydrogenase activity, cells were cultured in 96-well culture dishes and after incubation with the arsenic species for 24 and $48 \mathrm{~h}$, WST-8 (2-(2methoxy-4-nitrophenyl)-3-(4-nitrophenyl)-5-(2,4-disulfophenyl)$2 H$ tetrazolium, monosodium salt) solution was added and cells were incubated for a further hour. The absorbance in each well was measured using the plate reader at $450 \mathrm{~nm}$.

\section{Evaluation of monolayer and barrier integrity by TEER and capacitance measurement}

The human intestinal Caco-2 cell line is widely used as a model for the intestinal barrier ${ }^{41-43}$ and has been used previously to study transfer of arsenicals. ${ }^{33-36,44-47}$

Caco- 2 cells $\left(5 \times 10^{4}\right.$ cells per $\left.\mathrm{cm}^{2}\right)$ were seeded on Transwell ${ }^{\circledR}$ filter inserts with microporous polycarbonate membranes (1.12 $\mathrm{cm}^{2}$ growth area, $0.4 \mu \mathrm{m}$ pore size) adding $0.5 \mathrm{~mL}$ culture medium to the apical and $1 \mathrm{~mL}$ culture medium to the basolateral compartment.

The transepithelial electrical resistance (TEER) of the Caco-2 monolayer was used as a parameter for barrier integrity, measured using the cellZscope ${ }^{\circledR}$ (nanoAnalytics, Münster, Germany) device, suitable for a 12 Transwell $^{\circledR}$ filter system. The determined capacitance is directly proportional to the plasma membrane surface area; changes in the basolateral area and changes in the protein content and distribution may also contribute to capacitance changes. Only wells with TEER values $>1000 \Omega \mathrm{cm}^{2}$ and capacitance values between 3.8 and $5.0 \mu \mathrm{F} \mathrm{cm}^{-2}$, indicating a confluent monolayer with good barrier properties, were used for the experiments. Additionally, barrier integrity was evaluated by radiolabelled ${ }^{14} \mathrm{C}$-sucrose permeability. The main transport of sucrose is carried out via the paracellular route, since it is not transported actively or facilitated via the transcellular route. Thus, the amount of transported ${ }^{14} \mathrm{C}$-sucrose correlated directly with the tightness of the cell layer. Radiolabelled ${ }^{14} \mathrm{C}$-sucrose (Amersham, Buckinghamshire, UK) was added to the apical side of the Transwell ${ }^{\mathbb{R}}$ filter, crossover of ${ }^{14} \mathrm{C}$-sucrose to the basolateral side was measured time-dependently and permeability was calculated as described previously. ${ }^{48}$

\section{Arsenic species induced effects on and transfer across the Caco-2 barrier model}

To study the impact of the arsenic species on the barrier integrity as well as their transfer across the in vitro barrier, the differentiated Caco-2 monolayer was exposed on day 11 to arsenic species on the apical side (modelling the intestinal lumen in vivo). During the following $48 \mathrm{~h}$ of incubation, samples were taken from the apical and basolateral compartments for determination of arsenic content using electrothermal atomic absorption spectrometry (AAS), while the respective TEER and capacitance values were monitored online. Finally, arsenic transfer from one compartment to the other was expressed as permeability in \% (in relation to the applied concentration) and as concentration (in $\mu \mathrm{M}$ ) in the acceptor compartment. In the case of a time-dependent linear correlation, permeability coefficient in $\mathrm{cm} \mathrm{s}^{-1}$ was calculated. ${ }^{40}$ The permeability coefficients $\left(P_{\mathrm{c}}\right)$ were calculated by applying eqn (1) and (2).

$$
P=\left(\frac{\mathrm{d} C}{\mathrm{~d} t}\right)\left(\frac{V_{\mathrm{ac}}}{A C_{\mathrm{o}}}\right)
$$




$$
\frac{1}{P_{\mathrm{c}}}=\frac{1}{P_{\mathrm{c}+\mathrm{f}}}-\frac{1}{P_{\mathrm{f}}}
$$

$(\mathrm{d} C / \mathrm{d} t)$ is the flow $\left(\mu \mathrm{M} \mathrm{s}^{-1}\right)$ determined by the linear slope of the concentration in an acceptor compartment versus time plot. $V_{\text {ac }}$ is the volume of the acceptor compartment (apical: $0.5 \mathrm{~mL}$, basolateral: $1 \mathrm{~mL}$ ). $A$ is the growth area of the Transwell ${ }^{\mathbb{R}}$ $\left(1.12 \mathrm{~cm}^{2}\right)$ and $C_{\mathrm{o}}$ is the initial concentration of arsenic species in the donor compartment $\left(500 \mu \mathrm{M}\right.$ of oxo-DMAA ${ }^{\mathrm{V}}$, oxo-DMAE $^{\mathrm{V}}$, thio-DMAA $^{\mathrm{V}}$, thio-DMAE ${ }^{\mathrm{V}}, 1,2.5$ and $5 \mu \mathrm{M}$ of thio-DMA ${ }^{\mathrm{V}}$ and arsenite). $P_{\mathrm{f}}$ is the arsenic permeability of the Transwell ${ }^{\mathbb{R}}$ filter, $P_{\mathrm{c}+\mathrm{f}}$ the arsenic permeability of the filter in combination with the cell layer and $P_{\mathrm{c}}$ the arsenic permeability of the cell layer only (eqn (2)).

All studies were conducted in triplicate independent cultures. In the case of arsenite and thio-DMA ${ }^{\mathrm{V}}$ additional effects were investigated after incubation in the basolateral compartment as well as after simultaneous incubation on both sides.

\section{Cellular total arsenic concentrations}

Total arsenic concentrations were measured using electrothermal AAS after incubation of the cells with the respective arsenicals and subsequent wet ashing of cells. Due to the low number of cells used and the low arsenic concentrations applied in the barrier studies, we were not able to quantify the total arsenic content in cells grown on Transwell ${ }^{\mathbb{R}}$ filters. Instead, we used cells that were grown in culture dishes. Briefly, on day 11 cells were exposed to the respective arsenic compound for $24 \mathrm{~h}$ and $48 \mathrm{~h}$, trypsinized, collected by centrifugation and washed with ice-cold PBS; for standardization protein content was measured. Cell pellets were incubated for 15 min on ice with $300 \mu \mathrm{L}$ RIPAbuffer, sonicated on ice and centrifuged at $15000 \times g\left(4{ }^{\circ} \mathrm{C}\right)$ for $20 \mathrm{~min}$. The cellular protein level was quantified by the Bradford assay in an aliquot of the supernatant. ${ }^{12}$ Subsequently, the cell suspension was mixed and incubated with the ashing mixture $\left(65 \% \mathrm{HNO}_{3} / 30 \% \mathrm{H}_{2} \mathrm{O}_{2}(1 / 1)\right)$ at $95{ }^{\circ} \mathrm{C}$ for at least $12 \mathrm{~h}$. After dilution with bidistilled water, the arsenic concentration was determined using AAS as published before. ${ }^{37}$

\section{Metabolism of arsenite and thio-DMA ${ }^{\mathrm{V}}$ in the Caco-2 barrier model}

To study metabolism of arsenite and thio-DMA ${ }^{\mathrm{V}}$ in the Caco-2 intestinal barrier model, arsenite or thio-DMA ${ }^{\mathrm{V}}$ (each $2.5 \mu \mathrm{M}$ ) was applied to the apical side and the resulting species in the apical and basolateral compartments were measured using LC-ICP-QMS at several time points (i.e. 0, 6, 8, 10, 12, 14, 16, 24 and $48 \mathrm{~h}$ ) immediately after sampling. Therefore the sampled cell culture medium was directly injected into the injection loop of the autosampler without any further sample treatment to prevent species transformation. This was also the reason why the sample tray was cooled to $4{ }^{\circ} \mathrm{C}$ during analysis of the samples. To investigate the spontaneous (abiotic) formation of arsenic species, similar experiments were carried out in the absence of Caco- 2 cells.

The HPLC system consisted of a quaternary low pressure gradient LC pump (AccelaPump 1250 ${ }^{\mathrm{TM}}$, Thermo Fisher Scientific,
San Jose, CA, USA) and a corresponding autosampler (AccelaAutosampler $^{\mathrm{TM}}$, Thermo Fisher Scientific, San Jose, CA, USA), which was equipped with a 6-port stainless steel injection valve. For separation, a slight modification of a published procedure was used. $^{17}$ The method utilizes anion-exchange chromatography under isocratic conditions with a $20 \mathrm{mM}$ ammonium carbonate buffer adjusted to $\mathrm{pH} 8$ with ammonium formate as the mobile phase. Due to the volatile buffer system and the robust free-running generator of the ICP-QMS instrument, a large inner diameter of the injector pipe could be used, which further improved the limits of detection. Isocratic elution was sufficient to separate all the investigated species and to exhibit enough retention strength to move the species away from the void volume. The exit of the tempered $\left(40{ }^{\circ} \mathrm{C}\right)$ column was directly connected to the PFA nebulizer of the ICP-QMS, which was tested prior to the experiments regarding its

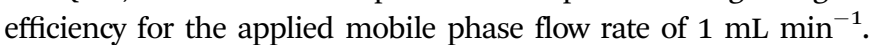
Further HPLC conditions are listed in Table 1; the partial loop filling is a compromise between maximum reproducibility and minimum biological sample volume needed. For quantification, calibration with a standard mix of $\mathrm{DMA}^{\mathrm{V}}$, monomethylarsonic acid, arsenite, arsenate and thio-DMA ${ }^{\mathrm{V}}$, diluted in culture medium, was applied (Fig. 7A). DMA ${ }^{\mathrm{III}}$ was measured as a separate standard, especially since this arsenical is not stable in aqueous solution (Fig. 7A). The automatically calculated peak areas were used for the external calibration and typical figures of merit could be obtained. In addition, blank values and sensitivity for a matrix calibration were compared to a standard calibration in bidistilled water and QA/QC samples (i.e. CCB as well as CCV) were measured each 25 injections.

For detection a highly sensitive ICP-QMS (iCAP Qc ${ }^{\mathrm{TM}}$, Thermo Fisher Scientific, Bremen, Germany), which was equipped with a novel flatapole collision/reaction cell $\left(\mathrm{QCell}^{\mathrm{TM}}\right.$ ) in the high sensitivity (2.8 $\mathrm{mm}$ Ni skimmer insert), kinetic energy discrimination (KED) mode was used. The plasma dependent parameters (i.e. torch position, nebulizer gas flow and potential of lenses located in front or directly after the right angle positive ion deflection lens) were tuned on a daily basis for maximum sensitivity (an oxide ratio of $<1.9 \%\left({ }^{140} \mathrm{Ce}^{16} \mathrm{O} /{ }^{140} \mathrm{Ce}^{+}\right.$) and a double charged ratio of $<3 \%\left({ }^{137} \mathrm{Ba}^{++} /{ }^{137} \mathrm{Ba}^{+}\right)$with background counts $<0.1 \mathrm{cps}(\mathrm{m} / z=4.5$ and 220.5) in standard mode of the instrument), while an appropriate bias potential of cell and quadrupole in conjunction with the flow rate of the cell gas was found by plotting the sensitivity of $1 \mu \mathrm{g} \mathrm{L} \mathrm{L}^{-1}{ }^{59} \mathrm{Co}$ against the interference ${ }^{35} \mathrm{Cl}^{16} \mathrm{O}$. The sufficient interference reduction by means of separation according to the axial kinetic energy after collisions with the inert cell gas was additionally investigated

Table 1 Parameters of the LC system

\begin{tabular}{ll}
\hline Parameter & Value \\
\hline LC & Thermo AccelaAS; AccelaPump \\
Column & Hamilton PRP-X 100; $4150 \mathrm{~mm}, 10 \mu \mathrm{m}$ \\
Elution & Isocratic $\left(1 \mathrm{~mL} \mathrm{~min}{ }^{-1}\right)$ \\
Mobile phase & $\left(\mathrm{NH}_{4}\right)_{2} \mathrm{CO}_{3}[20 \mathrm{mM}], \mathrm{pH} 8$ \\
Injection volume & $10 \mu \mathrm{L}($ partial loop) \\
Duration & $20 \mathrm{~min}$
\end{tabular}


Table 2 Conditions for iCAP Qc ICP-MS (Thermo Fisher Scientific)

\begin{tabular}{|c|c|}
\hline Parameter & Value \\
\hline Nebulizer gas & $\approx 0.9 \mathrm{~L} \mathrm{~min}^{-1}$ (daily tuned) \\
\hline Cell gas flow/KED barrier & $8 \%(\mathrm{v} / \mathrm{v}) \mathrm{H}_{2}$ in $\mathrm{He} @ 4.9 \mathrm{~mL} \mathrm{~min}{ }^{-1}, 3 \mathrm{~V}$ \\
\hline Isotope & ${ }^{75} \mathrm{As}$ \\
\hline LOQ (aqueous) (DIN 32645) & $0.08 \mu \mathrm{M}$ (arsenite); $0.05 \mu \mathrm{M}\left(\mathrm{DMA}^{\mathrm{V}}\right) ; 0.07$ (MMA ${ }^{\mathrm{V}}$ ); $0.05 \mu \mathrm{M}$ (thio-DMA $\mathrm{DA}^{\mathrm{V}}$ ); $0.13 \mu \mathrm{M}\left(\mathrm{iAs}^{\mathrm{V}}\right.$ ) \\
\hline LOQ (Matrix) (DIN 32645) & $0.28 \mu \mathrm{M}$ (arsenite); $0.09 \mu \mathrm{M}\left(\mathrm{DMA}^{\mathrm{V}}\right) ; 0.05\left(\mathrm{MMA}^{\mathrm{V}}\right) ; 0.34 \mu \mathrm{M}($ thio-DMA $) ; 0.10 \mu \mathrm{M}\left(\mathrm{iAs}^{\mathrm{V}}\right)$ \\
\hline
\end{tabular}

by the introduction of different $\mathrm{HCl}$ concentrations in the range of $10 \mathrm{ppm}$ to $0.1 \%$, whereas no significant increase of the baseline on $\mathrm{m} / \mathrm{z} 75$ could be found. Together with the non-significant differences between the standard and the matrix calibration, this puts further evidence on the accuracy of the applied method. Important parameters of the ICP-QMS instrument are listed in Table 2.

\section{Immunocytochemistry}

Immunocytochemistry studies were conducted in Caco-2 monolayers grown on Transwell ${ }^{\circledR}$ filters as described previously. ${ }^{40}$ As primary antibody, mouse anti-occludin $\left(1 \mu \mathrm{g} \mathrm{mL} L^{-1}\right)$ was incubated at $37{ }^{\circ} \mathrm{C}$ in a BSA solution $(0.5 \%)$ for $30 \mathrm{~min}$. The secondary antibody alexa fluor ${ }^{\mathbb{R}} 546$ goat anti-mouse $\left(1 \mu \mathrm{g} \mathrm{mL}{ }^{-1}\right)$ was diluted in BSA (0.5\%) and applied for $1 \mathrm{~h}$ at $37^{\circ} \mathrm{C}$. Furthermore, cell nuclei were stained with Hoechst $33258\left(10 \mu \mathrm{g} \mathrm{mL} \mathrm{m}^{-1}\right)$ for $0.5 \mathrm{~min}$. Filters with immunostained cells were cut out from the inserts, and mounted in Aqua Poly/Mount. Microscopic images were taken using the fluorescence microscope Axio ImagerM2 (Zeiss, Oberkochen, Germany) and evaluated using Axiovision 4.5 software (Zeiss, Göttingen, Germany).

\section{Results}

In this study, the impact of $500 \mu \mathrm{M}$ of oxo-DMAA ${ }^{\mathrm{V}}$, oxo-DMAE ${ }^{\mathrm{V}}$, thio-DMAA ${ }^{\mathrm{V}}$ and thio-DMAE ${ }^{\mathrm{V}}$ on the Caco-2 intestinal barrier model as well as their transfer across this barrier were measured. Furthermore, cytotoxicity, cellular uptake, presystemic metabolism and intestinal transfer of arsenite and thio-DMA ${ }^{\mathrm{V}}$ were assessed.

\section{Monitoring differentiation}

Cells reached a differentiation state around 8-9 days after seeding as monitored by transepithelial electrical resistance (TEER) and capacitance (Fig. 2A), being tracers for barrier integrity and the membrane surface, respectively. During the first days after seeding, TEER and capacitance increased until reaching a steady state level after days 8-9. After day 16 of cultivation, a decrease in TEER was observed. The barrier properties of the Caco-2 cell monolayer were further investigated by the immunocytochemical analysis of occludin, a protein that is a central functional component of tight junctions. On day 11 after seeding, occludin staining appeared in clear lines, without any fuzzy appearance, and cell borders were mostly straight and not serrated (Fig. 2B). Moreover, comparative transfer experiments were carried out by incubating differentiated Caco-2 cells 11 and 21 days after seeding with $5 \mu \mathrm{M}$ arsenite in the apical



Fig. 2 Online monitored development of transepithelial electrical resistance (TEER) and capacitance values from day 2 after seeding of Caco-2 cells on Transwell ${ }^{\mathbb{B}}$ filters until day 21 of cultivation (A). Immunocytochemical labeling of the tight junction protein occludin in differentiated Caco-2 cells 11 days after seeding (B). Shown are data from a representative experiment.

compartment. Here, similar transfer rates were achieved (data not shown). Accordingly, all further transfer experiments were carried out with cells in passage number 53 on day 11 after initial seeding.

During all transfer experiments, barrier integrity was permanently monitored by online measurement of TEER and capacitance; in addition ${ }^{14} \mathrm{C}$-sucrose permeability was determined. The limits established for intact barriers were: TEER $>1000 \Omega \mathrm{cm}^{2}$; capacitance around 3.8-5.0 $\mu \mathrm{F} \mathrm{cm}^{-2}$ and a ${ }^{14} \mathrm{C}$-sucrose permeability coefficient $\leq 0.2 \times 10^{-6} \mathrm{~cm} \mathrm{~s}^{-1}$. If changes in TEER and capacitance of more than $30 \%$ in comparison to untreated control cells were observed, disruption of barrier integrity was recorded.

\section{Impact of the arsenicals on barrier integrity and viability of Caco-2 cells}

The online measurement of TEER (Fig. 3A, C and E) and the electrical capacitance (Fig. 3B, D and F) confirmed an intact barrier after apical incubation with 1-5 $\mu \mathrm{M}$ of thio-DMA ${ }^{\mathrm{V}}$ (Fig. 3A and B), 1-5 $\mu \mathrm{M}$ of arsenite (Fig. 3C and D) and $500 \mu \mathrm{M}$ of oxo-DMAA ${ }^{\mathrm{v}}$, oxo-DMAE ${ }^{\mathrm{V}}$, thio-DMAA ${ }^{\mathrm{V}}$ or thio-DMAE ${ }^{\mathrm{V}}$ (Fig. $3 \mathrm{E}$ and F). Higher oxo-DMAA ${ }^{\mathrm{V}}$, oxo-DMAE ${ }^{\mathrm{V}}$, thio-DMAA ${ }^{\mathrm{V}}$ or thio$\mathrm{DMAE}^{\mathrm{V}}$ concentrations are unlikely to be exposure relevant and were therefore not applied. Since in the case of arsenite and thio-DM ${ }^{\mathrm{v}}$, TEER and capacitance values were the most sensitive tested cellular toxicity markers, effects of oxo-DMAA ${ }^{\mathrm{V}}$, oxo$\mathrm{DMAE}^{\mathrm{V}}$, thio-DMAA ${ }^{\mathrm{V}}$ or thio-DMAE ${ }^{\mathrm{V}}$ on additional toxicity markers were not tested.

Arsenite affected TEER and capacitance faster as compared to thio-DMA ${ }^{\mathrm{V}}$ and caused an irreversible disruption of the barrier after apical incubation with $10 \mu \mathrm{M}$; comparable effects 

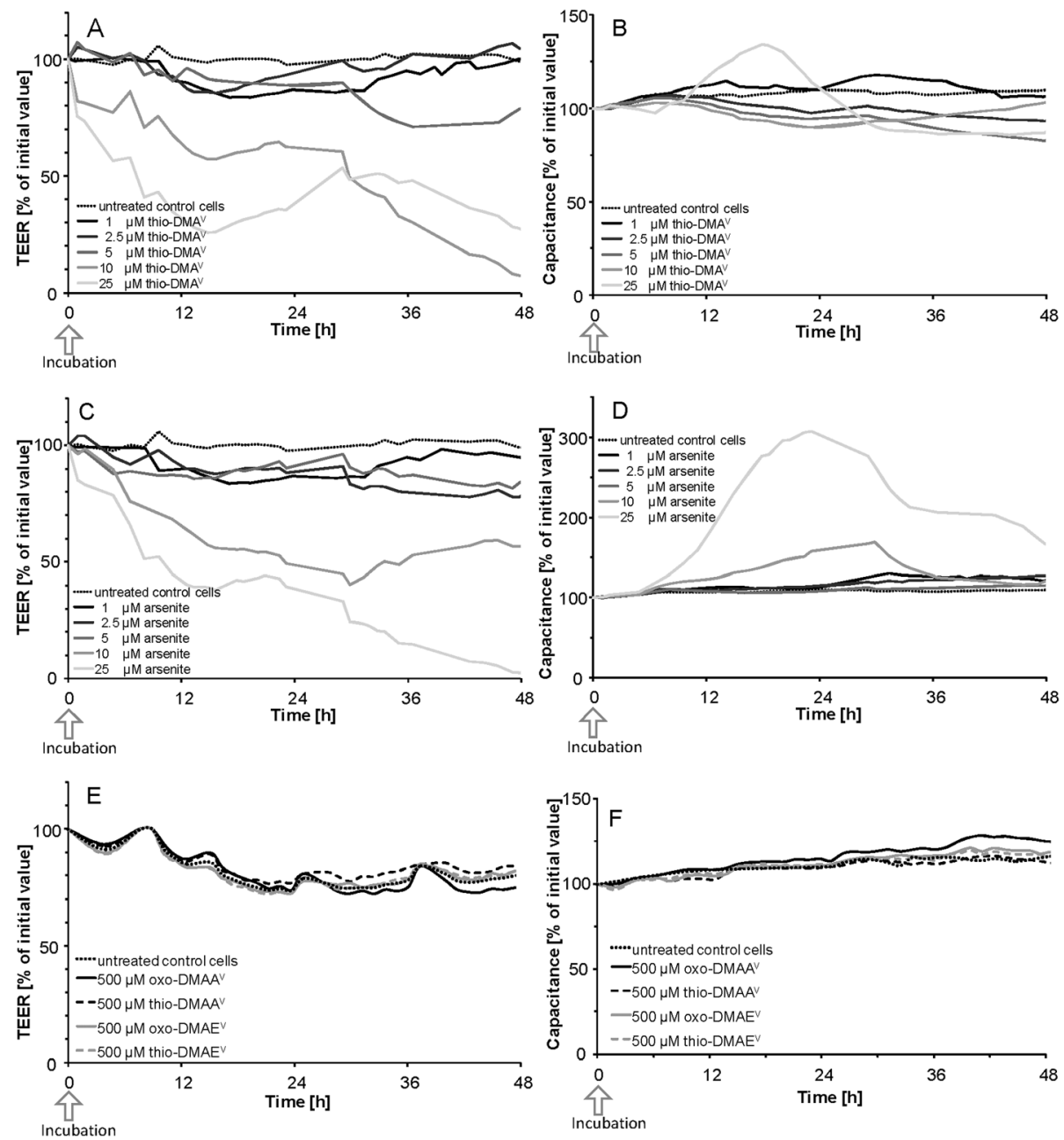

Fig. 3 TEER ( $A, C, E)$ and capacitance values $(B, D$ and $F$ ) after apical incubation of the Caco-2 barrier with thio-DMA $(A$ and $B)$, arsenite (C and $D)$ and oxo-DMAA oxo-DMAE ${ }^{\vee}$, thio-DMAA $A^{\vee}$ and thio-DMAE ${ }^{\vee}(E$ and F) data, expressed as \% of an initial value; shown are representative measurements. Absolute TEER and capacitance were on average $1600 \Omega \mathrm{cm}^{2}$ and $4.5 \mu \mathrm{F} \mathrm{cm}^{-2}$, respectively.

were achieved by $25 \mu \mathrm{M}$ thio-DMA ${ }^{\mathrm{V}}$. In contrast, the measured cellular viability endpoint lysosomal integrity (Fig. 4A) and cellular dehydrogenase activity (Fig. 4B) indicate stronger effects by thio-DMA ${ }^{\mathrm{V}}$.

To guarantee barrier integrity and thus to avoid barrier leakage, transfer experiments were carried out with a maximum of $5 \mu \mathrm{M}$ arsenite or thio-DMA ${ }^{\mathrm{V}}$. Further studies demonstrated that even after basolateral incubation and simultaneous incubation in both compartments, barrier integrity was not affected by these arsenic species concentrations (data not shown).

\section{Permeability of oxo-DMAA ${ }^{\mathrm{v}}$, oxo-DMAE ${ }^{\mathrm{v}}$, thio-DMAA ${ }^{\mathrm{v}}$, thio-DMAE ${ }^{\mathrm{V}}$ after apical incubation}

Intestinal bioavailability of the metabolites (each $500 \mu \mathrm{M}$ ) was assessed by measuring their time-dependent crossover after apical incubation in the Caco-2 barrier model, while online monitoring barrier integrity. Here, the differentiated Caco-2 cells grown on Transwell ${ }^{\mathbb{R}}$ filters build a two chamber model, with the cell layer resembling the intestinal barrier, the upper apical side referring to the intestinal lumen and the lower basolateral side referring to the blood side. For better understanding, the results are given expressed in terms of arsenic concentrations in the acceptor (basolateral) compartment (Table 3A) and as percentage permeability in relation to the applied concentration (Table 3B). In addition, the permeability coefficients were calculated (Table 3C). Transfer rates of oxo-DMAA ${ }^{\mathrm{V}}$, oxo-DMAE ${ }^{\mathrm{V}}$ and thio-DMAA ${ }^{\mathrm{V}}$ were quite small, amounting to about $2-5 \%$ after $48 \mathrm{~h}$ of incubation. In contrast thio-DMAE ${ }^{\mathrm{V}}$ crossover was very efficient reaching a percentage permeability of $54.5 \pm 3.0 \%$ and thus an equilibrium of concentration in both compartments after $48 \mathrm{~h}$ incubation.

\section{Permeability of arsenite and thio-DMA}

To investigate transfer of arsenite and thio-DMA ${ }^{\mathrm{V}}$ in detail, incubation was carried out with sub-cytotoxic concentrations on the apical side, on the basolateral side or on both sides simultaneously, while barrier integrity (TEER and capacitance) was monitored during the entire experiments. Accordingly, ${ }^{14} \mathrm{C}$-sucrose permeability was not affected by these concentrations (Fig. 5A). The result of the transfer experiments from 


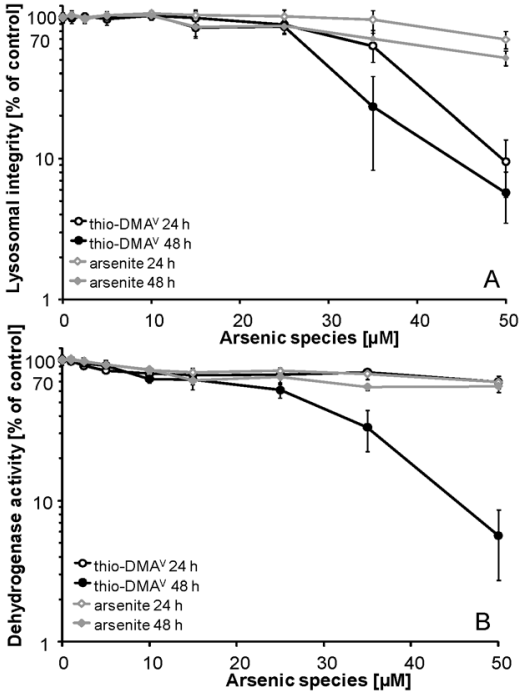

Fig. 4 Cytotoxicity of arsenite and thio-DMA ${ }^{\vee}$ in Caco-2 cells after 24 and $48 \mathrm{~h}$ incubation. Cytotoxicity was determined by a decrease in lysosomal integrity (A) and cellular dehydrogenase activity (B), as measured by the neutral red uptake assay and the CCK- $8{ }^{\mathbb{R}}$ assay, respectively. The data represent mean values of at least 4 determinations \pm SD.

the apical to basolateral side is shown in the respective figures expressed as permeability coefficient (Fig. 5B), in terms of arsenic concentrations in the acceptor compartment (as percentage normalized to the applied dose) (Fig. 5C and D) and as percentage permeability in relation to the applied concentration (Fig. 5E and F).

In summary, the crossover behaviour of both species is similar and seems to be independent of the applied concentrations. Thus, after $24 \mathrm{~h}$ incubation, comparable arsenic concentrations were obtained in both compartments (Fig. 5C and D). Taking a closer look, after apical thio-DMA ${ }^{\mathrm{V}}$ incubation, especially in the first six hours, arsenic concentrations decreased more than after apical incubation with arsenite (Fig. 5E and F). Crossover after basolateral incubation with the arsenicals was comparable to their crossover after apical incubation (data not shown). Our data clearly show that in the observed concentration range (1-5 $\mu \mathrm{M})$, arsenic basolateral-toapical transfer was indistinguishable from the apical-to-basolateral transfer. This indicates that in the case of arsenite and thio-DMA ${ }^{\mathrm{V}}$, after incubation on the basolateral or the apical side, arsenic crossed the barrier in both directions (apical-to-basolateral and basolateral-to-apical transfer) to a comparable extent.

In a third approach, thio-DMA ${ }^{\mathrm{V}}$ or arsenite was incubated simultaneously in equal concentrations both in the apical and the basolateral medium. No accumulation was visible in either of the compartments within $48 \mathrm{~h}$ of incubation with $2.5 \mu \mathrm{M}$ arsenite or thio-DMA ${ }^{\mathrm{V}}$ (Fig. 5G and $\mathrm{H}$ ), pointing to an undirected arsenic species transfer process.

The described crossover behavior of arsenite and thio-DMA ${ }^{\mathrm{V}}$ is also reflected in the calculated permeability coefficients and the efflux ratio (Fig. 5B). The efflux ratio is calculated as the quotient of the $P_{\mathrm{c}}(\mathrm{b} \rightarrow \mathrm{a}) / P_{\mathrm{c}}(\mathrm{a} \rightarrow \mathrm{b})$. Efflux values $>1$ indicate increased efflux while values $<1$ indicate increased influx. Values around 1, as shown in our studies (Fig. 5B), suggest an equilibrium between influx and efflux.

\section{Cellular arsenic concentration after incubation with arsenite or thio-DMA}

Cellular total arsenic concentrations were determined after 24 and $48 \mathrm{~h}$ incubation with $1,2.5,5$, and $10 \mu \mathrm{M}$ arsenite or thio-DMA ${ }^{\mathrm{V}}$ (Fig. 6). Interestingly, for both species a longer

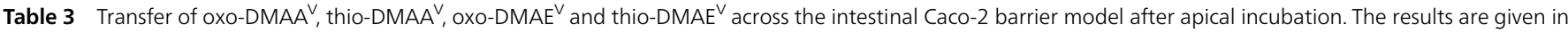

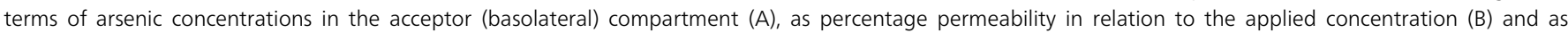
permeability coefficient (C). A factor two caused by volume differences between apical ( $0.5 \mathrm{~mL})$ and basolateral (1 $\mathrm{mL}) \mathrm{compartment}$ is considered

(A) Arsenic concentrations in the acceptor (basolateral) compartment $[\mu \mathrm{M}]$

\begin{tabular}{|c|c|c|c|c|}
\hline Time $[\mathrm{h}]$ & oxo-DMAA $^{\mathrm{V}}$ & thio-DMAA $^{\mathrm{V}}$ & oxo-DMAE ${ }^{\mathrm{V}}$ & thio-DMAE $^{\mathrm{V}}$ \\
\hline 0 & n.d. & n.d. & n.d. & n.d. \\
\hline 6 & $0.57 \pm 0.15$ & $1.85 \pm 0.26$ & $0.89 \pm 0.19$ & $27.3 \pm 0.47$ \\
\hline 12 & $1.06 \pm 0.13$ & $3.32 \pm 0.24$ & $2.59 \pm 0.40$ & $54.9 \pm 6.34$ \\
\hline 24 & $2.39 \pm 0.41$ & $6.30 \pm 0.36$ & $5.75 \pm 0.54$ & $93.4 \pm 13.1$ \\
\hline 36 & $3.36 \pm 0.60$ & $9.04 \pm 0.62$ & $10.2 \pm 1.06$ & $121 \pm 12.5$ \\
\hline 48 & $5.09 \pm 1.33$ & $8.54 \pm 4.16$ & $12.2 \pm 2.88$ & $136 \pm 7.49$ \\
\hline
\end{tabular}

(B) Percentage permeability [\%] in relation to the applied concentration $[500 \mu \mathrm{M}]$

\begin{tabular}{|c|c|c|c|c|}
\hline Time $[\mathrm{h}]$ & oxo-DMAA $^{\mathrm{V}}$ & thio-DMAA $^{\mathrm{V}}$ & oxo-DMAE ${ }^{\mathrm{V}}$ & thio-DMAE $^{\mathrm{V}}$ \\
\hline 0 & n.d. & n.d. & n.d. & n.d. \\
\hline 6 & $0.23 \pm 0.06$ & $0.74 \pm 0.10$ & $0.36 \pm 0.08$ & $10.9 \pm 0.19$ \\
\hline 12 & $0.42 \pm 0.05$ & $1.33 \pm 0.10$ & $1.04 \pm 0.16$ & $22.0 \pm 2.54$ \\
\hline 24 & $0.96 \pm 0.16$ & $2.52 \pm 0.14$ & $2.30 \pm 0.21$ & $37.4 \pm 5.25$ \\
\hline 36 & $1.35 \pm 0.24$ & $3.62 \pm 0.25$ & $4.06 \pm 0.42$ & $48.3 \pm 4.98$ \\
\hline 48 & $2.04 \pm 0.53$ & $3.42 \pm 1.67$ & $4.88 \pm 1.15$ & $54.5 \pm 3.00$ \\
\hline
\end{tabular}

(C) Permeability coefficient $\left[\mathrm{cm} \mathrm{s}^{-1}\right]$

\begin{tabular}{|c|c|c|c|c|}
\hline$P_{\mathrm{c}}$ & oxo-DMAA $^{\mathrm{V}}$ & thio-DMAA ${ }^{\mathrm{V}}$ & oxo-DMAE ${ }^{\mathrm{V}}$ & thio-DMAE ${ }^{\mathrm{V}}$ \\
\hline & $0.05 \times 10^{-6}$ & $0.12 \times 10^{-6} \pm 0.01 \times 10^{-6}$ & $0.13 \times 10^{-6} \pm 0.03 \times 10^{-6}$ & $1.66 \times 10^{-6} \pm 0.21 \times 10^{-6}$ \\
\hline
\end{tabular}



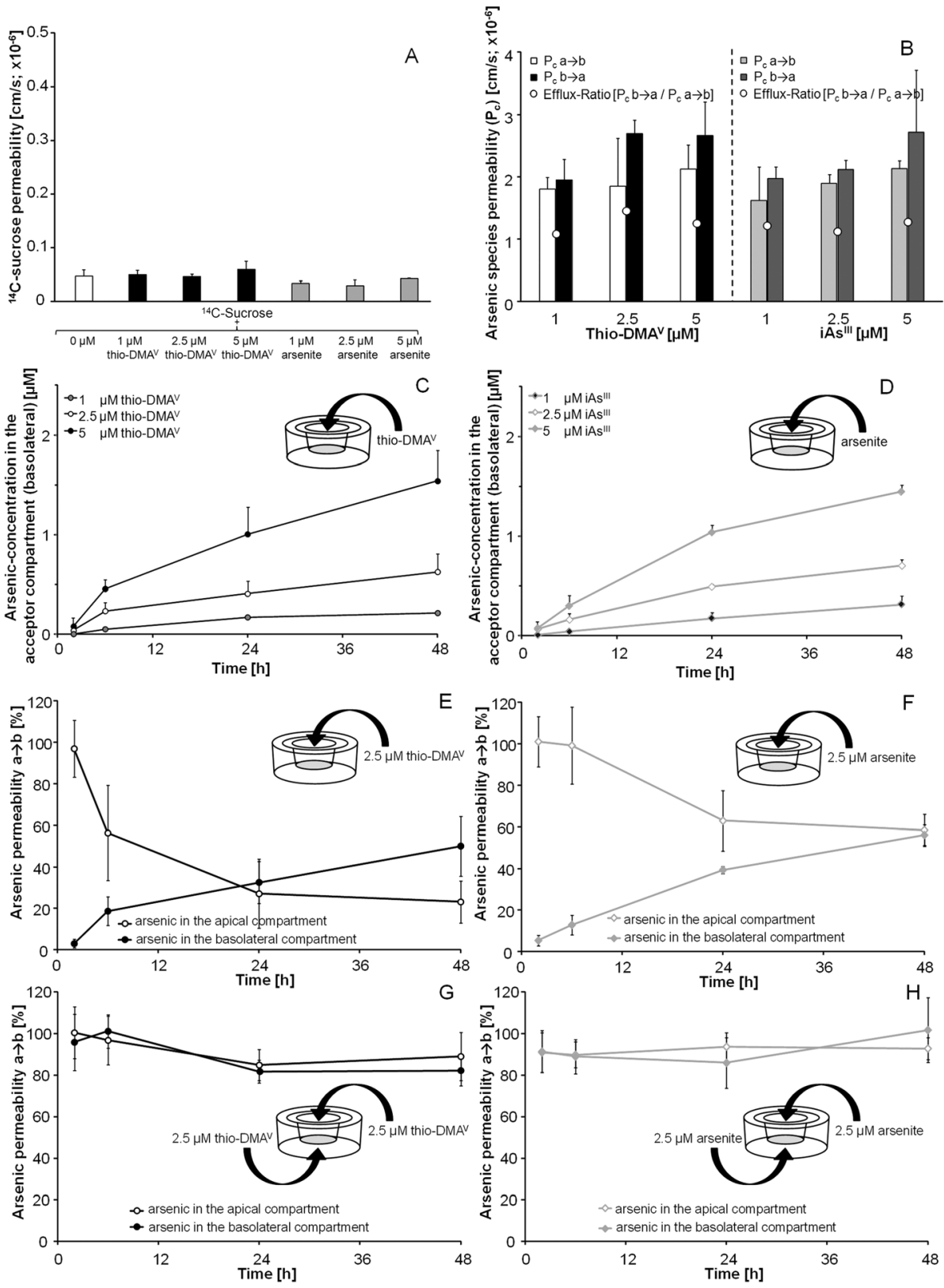

Fig. $5{ }^{14} \mathrm{C}$-sucrose permeability $(A)$ and arsenic species permeability (B), transfer of thio-DMA $(C$ and $E)$ and arsenite (D and F) across the intestinal Caco-2 barrier model after apical (a) incubation and transfer of thio-DMA ${ }^{\vee}(G)$ and arsenite $(\mathrm{H})$ after incubation on both sides simultaneously. Displayed are arsenic concentrations in the acceptor (basolateral (b)) compartment $[\mu \mathrm{M}](\mathrm{C}$ and $\mathrm{D})$ and percentage arsenic permeability (normalized to initial incubated concentration $(2.5 \mu \mathrm{M}))(\mathrm{E}, \mathrm{F}, \mathrm{G}$ and $\mathrm{H})$. Because of the volume differences in the apical $(0.5 \mathrm{~mL})$ and basolateral $(1 \mathrm{~mL})$ compartments a correction factor of two for the transfer has to be considered. The data represent mean values of at least 3 independent determinations, $+/ \pm$ SD.

incubation period did not affect cellular arsenic concentrations. Caco-2 cells incubated with thio-DMA ${ }^{\mathrm{V}}$ accumulate 6-8-fold more arsenic than after incubation with arsenite.

\section{Metabolism of arsenite and thio-DMA ${ }^{\mathrm{V}}$ by Caco-2 cells}

To assess metabolism of arsenite and thio-DMA ${ }^{\mathrm{V}}$ by the in vitro intestinal barrier $0,6,8,10,12,14,16,24$ and $48 \mathrm{~h}$ after incubation, samples were taken from the apical and basolateral side and arsenic species were analysed using LC-ICP-QMS. Analysis of a standard mixture of $\mathrm{DMA}^{\mathrm{v}}, \mathrm{MMA}^{\mathrm{v}}$, arsenite, arsenate and thio-DMA ${ }^{\mathrm{V}}$ and additionally a separate $\mathrm{DMA}^{\mathrm{III}}$ standard identification of detected peaks were carried out (Fig. 7A). In the case of apical incubation with arsenite, no arsenic metabolites were detected during the complete experiment in either the apical or the basolateral medium (Fig. 7C). In contrast, after apical thio-DMA $\mathrm{DA}^{\mathrm{V}}$ incubation, the metabolite $\mathrm{DMA}^{\mathrm{V}}$, in addition to thio-DMA $\mathrm{DA}^{\mathrm{V}}$, was identified in both the apical and the basolateral compartments (Fig. 7B).

Within $24 \mathrm{~h}$ incubation, about $31 \%$ of thio-DMA ${ }^{\mathrm{V}}$ was transformed in the Caco-2 in vitro barrier model into $\mathrm{DMA}^{\mathrm{V}}$ (Fig. 7D). In the absence of Caco- 2 cells, only $\approx 11 \%$ of thio$\mathrm{DMA}^{\mathrm{V}}$ was transformed into $\mathrm{DMA}^{\mathrm{V}}$, presumably because of 


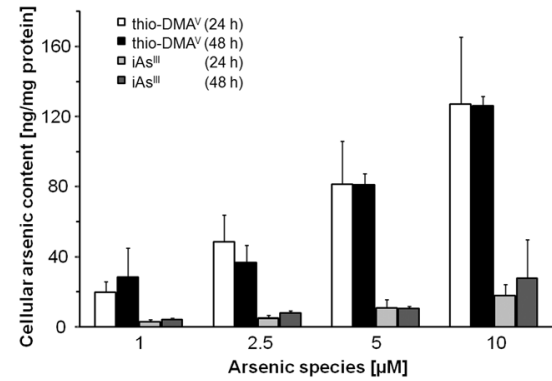

Fig. 6 Cellular bioavailability of arsenite and thio-DMA $\vee$ after 24 and $48 \mathrm{~h}$ incubation of differentiated Caco-2 cells. Shown are mean values of at least 3 independent determinations $+\mathrm{SD}$; arsenic concentration in untreated control cells was below the detection limit $\left(<0.2 \mathrm{ng} \mathrm{mg}^{-1}\right)$.
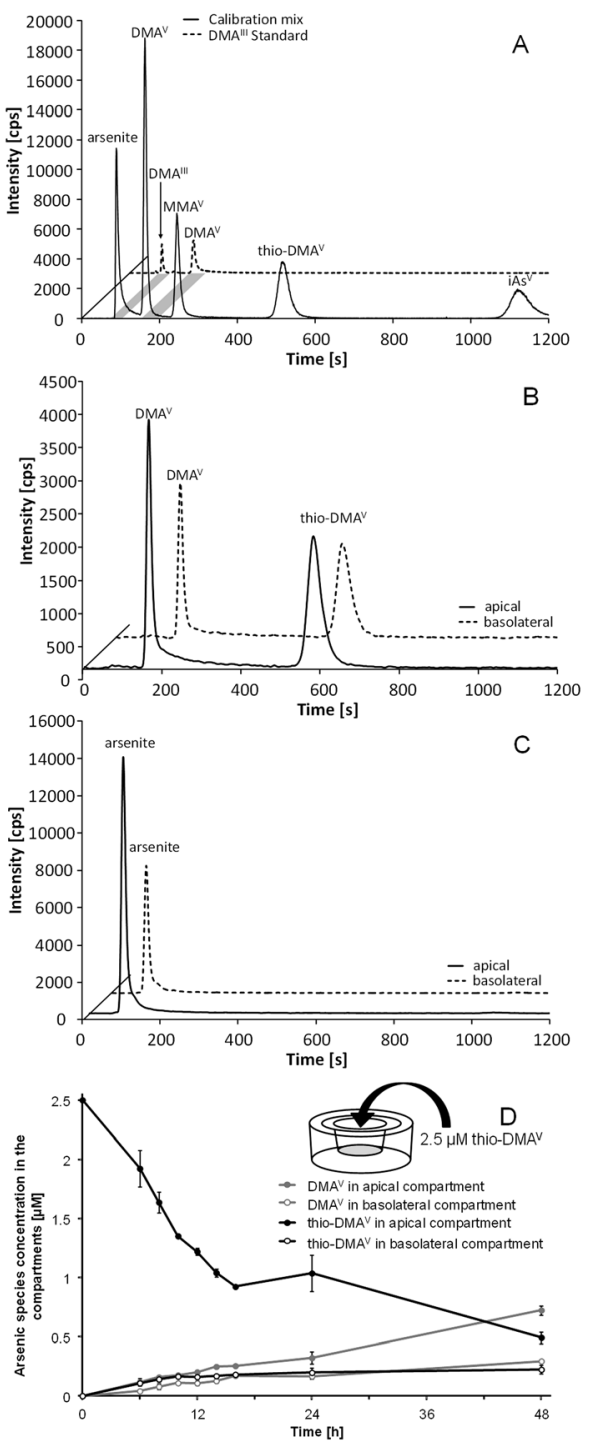

Fig. 7 Speciation of arsenic species during $48 \mathrm{~h}$ transfer experiments. Transfer and resulting species after apical incubation with $2.5 \mu \mathrm{M}$ thio-DMA $\mathrm{A}^{\mathrm{V}}$ or arsenite as observed using LC-ICP-QMS. Displayed are representative chromatograms of a standard calibration solution as well as DMA"I' standard (A), chromatograms of apical (a) as well as basolateral (b) samples after thio-DMA ${ }^{\mathrm{V}}$ (B) and arsenite (C) incubation $(16 \mathrm{~h})$. Furthermore the progress of thio-DMA $\mathrm{A}^{\mathrm{V}}$ transfer and metabolism (D) is shown; the data represent mean values of at least 3 independent determinations, $\pm S D$. dissolved oxygen in the media. This indicates that the remaining formation of $20 \% \mathrm{DMA}^{\mathrm{V}}$ resulted from metabolism by the cells either directly or indirectly.

\section{Discussion}

Whereas inorganic arsenic is classified as a human carcinogen, ${ }^{2,3}$ risks to human health related to the presence of arsenosugars in marine food are still unclear. ${ }^{1}$ Promising recent approaches to further understand inorganic arsenic induced carcinogenicity include the characterization of the toxicity of inorganic arsenic metabolites and the assessment of their contribution to the carcinogenic process. Because several human inorganic arsenic metabolites have been shown to exert greater toxicity than arsenite, and thus are likely to strongly contribute to inorganic arsenic induced carcinogenicity, a risk assessment for arsenosugars should also include a toxicological characterization of their respective metabolites.

Recent studies indicate that after the ingestion of food containing organic and inorganic arsenic species, these species can be partially degraded or biotransformed by presystemic metabolism. ${ }^{28,47,49}$ Additionally, various inorganic and organic arsenic metabolites might reach the GI tract through the bile secretion. ${ }^{26,50,51}$ This has been postulated before for thio-DMA ${ }^{\mathrm{V}}$, which is also a metabolite of arsenosugars. ${ }^{26}$ Thus similar effects cannot be excluded for other arsenosugar metabolites. Indeed, when taking into account the observed extensive metabolism of arsenosugars in humans, ${ }^{5,21,22}$ and the recently observed low intestinal bioavailability of arsenosugars as assessed by the Caco2 model, ${ }^{29}$ formation of arsenosugar metabolites prior to intestinal absorption seems to be likely. In the present study we assessed intestinal bioavailability of the human arsenosugar metabolites oxo-DMAA ${ }^{\mathrm{V}}$, thio-DMAA ${ }^{\mathrm{V}}$, oxo-DMAE ${ }^{\mathrm{V}}$, thio-DMAE ${ }^{\mathrm{V}}$ and thio-DMA ${ }^{\mathrm{V}}$ in relation to arsenite. ${ }^{5,21,22}$

After apical incubation with the respective species, only arsenite and thio-DMA ${ }^{\mathrm{V}}$ caused a barrier disruption, which has been shown for arsenite before. ${ }^{44}$ Nevertheless, the respective concentrations $(\geq 10 \mu \mathrm{M})$ necessary to cause this disruption are unlikely to be exposure relevant. All other metabolites did not cause a barrier leakage, even when applied at 50 times higher concentrations than arsenite and thio-DMA $\mathrm{v}$. Accordingly, in the two studies available, these metabolites exerted only minor in vitro toxicity. ${ }^{22,29}$

Comparing the toxic effects on the in vitro intestinal barrier induced by arsenite and thio-DMA $\mathrm{V}$, and taking into account the respective determined cellular arsenic contents, arsenite seems to have higher toxic potential than thio-DMA ${ }^{\mathrm{V}}$. Cellular arsenic content in differentiated Caco-2 cells was about 6-8-fold higher after incubation with thio-DMA ${ }^{\mathrm{V}}$ than after incubation with equivalent arsenite concentrations. Therefore, toxic effects were elicited by much lower effective cellular arsenic concentrations in the arsenite incubations. Nevertheless, as a pentavalent arsenic species, thio$\mathrm{DMA}^{\mathrm{V}}$ exerts relatively strong cellular toxicity, which might be partly explained by the generation of trivalent, even more toxic DMA ${ }^{\mathrm{III}}$ or DMAG $^{\text {III }}$ inside the cells. ${ }^{52}$

Regarding the influx transfer, after $48 \mathrm{~h}$ incubation thio$\mathrm{DMA}^{\mathrm{V}}$ and thio-DMAE ${ }^{\mathrm{V}}$ exerted similar transfer rates $(\approx 50 \%)$ 
as compared to arsenite, thereby reaching a concentration equilibrium in both compartments. In contrast, oxo-DMAA ${ }^{\mathrm{V}}$, thio-DMAA ${ }^{\mathrm{V}}$, oxo-DMAE ${ }^{\mathrm{V}}$ passed the intestinal barrier only to a very small extent. The low permeability of oxo-DMAA ${ }^{\mathrm{V}}$, thio$\mathrm{DMAA}^{\mathrm{V}}$, oxo-DMAE ${ }^{\mathrm{V}}$ allows the conclusion that these species did not pass the barrier by the transcellular route at the applied $\mathrm{pH}$ of 6.8. Nevertheless, in vivo a paracellular transport might be possible, since in the Caco- 2 model this route is often underestimated because of the strong tightness of tight junctions. In vivo tight junctions of the intestinal mucosa are much more permeable. ${ }^{30,32,53}$ The observed differences in intestinal crossover might be attributed to different molecule polarity of the respective arsenic species. Thus, $\mathrm{p} K_{\mathrm{a}}$ values of the molecules might explain absorption properties. At the applied $\mathrm{pH}$ in culture media uncharged molecules, including arsenite, would show a better crossover than charged molecules. ${ }^{8}$

The calculated permeability coefficients for thio-DMA ${ }^{\mathrm{V}}$, thio$\mathrm{DMAE}^{\mathrm{V}}$ and arsenite for the absorptive route are $1.85 \pm 0.77 \times$ $10^{-6} \mathrm{~cm} \mathrm{~s}^{-1}, 1.66 \pm 0.21 \times 10^{-6} \mathrm{~cm} \mathrm{~s}^{-1}$ and $1.89 \pm 0.15 \times$ $10^{-6} \mathrm{~cm} \mathrm{~s}^{-1}$, respectively. The permeability coefficient for arsenite fits well to the published data. Thus, other studies predicted for arsenite absorption permeability coefficients between 1.1-4.6 $\times 10^{-6} \mathrm{~cm} \mathrm{~s}^{-1}$ across paracellular (tight junctions) and transcellular pathways. ${ }^{33-35}$ The high permeability of thio-DMA ${ }^{\mathrm{V}}$ and thio-DMAE ${ }^{\mathrm{V}}$ is remarkable and has not been shown before. All other pentavalent arsenicals investigated so far show about a 10fold lower intestinal bioavailability than arsenite and are assumed to be transferred mainly via the paracellular route. ${ }^{34,36}$ Despite their pentavalence both arsenic species, like arsenite, seem to cross the intestinal barrier via para- and transcellular routes, probably via similar transport modes. Nevertheless, since only thio-DMA ${ }^{\mathrm{V}}$ exerted effects on barrier disruption at low concentrations and has been shown before to be a potent toxic arsenic species, we decided to focus on thio-DMA $\mathrm{V}$. In a next step, we further characterized its intestinal bioavailability thereby taking also into account its metabolism in the in vitro system.

Efflux and influx of arsenic across the intestinal barrier model were comparable after incubation with thio-DMA $\mathrm{D}^{\mathrm{V}}$ and arsenite. After simultaneous incubation of equal concentrations of thio-DMA ${ }^{\mathrm{V}}$ or arsenite on both sides, no active driven secretory process or accumulation at a preferred side was detected. This indicates a concentration gradient driven crossover. Thus arsenite and thio-DMA ${ }^{\mathrm{V}}$ crossed the intestinal barrier most likely by passive diffusion (paracellular) and facilitated (transcellular) transport. Accordingly, for arsenite a balance between a saturable intestinal influx transport system and a secretory system has been proposed before. ${ }^{35}$ Detailed studies reported the OATPB, AQP10, GLUT5 to be the most likely transporters for transcellular transport of arsenite. ${ }^{46}$ These bidirectional transporters might control both the cellular influx and the efflux of arsenite. For thio-DMA ${ }^{\mathrm{V}}$ no such studies exist so far. When directly comparing arsenic transfer after incubation with arsenite and thio-DMA ${ }^{\mathrm{V}}$, obviously in the case of thio-DMA ${ }^{\mathrm{V}}$ the decrease of arsenic in the apical compartment (representing the intestinal lumen) was faster than in the case of arsenite incubation. Differences in arsenic concentrations were not that obvious in the target compartment, which might be partly due to a massive accumulation of arsenic in the cells of the barrier after incubation with thio-DMA $\mathrm{V}$.

Since a recent study applying hydride generation-cold trapatomic absorption spectrometry indicates that under certain conditions differentiated Caco- 2 cells can metabolize inorganic arsenic to methylated arsenic species, ${ }^{47}$ we carried out LC-ICPQMS based arsenic speciation studies during the transfer experiments. In the case of arsenite incubation, over the whole period of the experiment $(0-48 \mathrm{~h})$, we detected no arsenic species other than arsenite both in the donor and the acceptor compartment. Our analytical method (LC-ICP-QMS) provides selective and sensitive quantification of arsenite, arsenate, $\mathrm{DMA}^{\mathrm{V}}$, thio-DMA ${ }^{\mathrm{V}}, \mathrm{DMA}^{\mathrm{III}}$ and $\mathrm{MMA}^{\mathrm{V}}$ in the respective compartments. Although we cannot exclude the possibility that other arsenic metabolites are formed that co-eluate with arsenite, we can conclude that under our experimental conditions differentiated Caco-2 cells do not metabolize arsenite and subsequently secrete arsenate, $\mathrm{DMA}^{\mathrm{V}}, \mathrm{MMA}^{\mathrm{V}}, \mathrm{DMA}^{\mathrm{III}}$ or thio-DMA ${ }^{\mathrm{V}}$ in detectable concentrations. This has also been reported before by Laparra et al. ${ }^{35}$ The observed different outcome in comparison to Calatayud et al., 2012 might be due to differences in the applied arsenite concentrations and cell culture conditions; ${ }^{47}$ additionally Caco- 2 cells were supplied from different sources and might thereby exert different metabolic capacities. Moreover, different analytical techniques were used to identify and quantify arsenic species.

In contrast to arsenite, thio-DMA ${ }^{\mathrm{V}}$ was metabolized by the applied in vitro intestinal barrier system. On both sides of the Caco-2 barrier, thio-DMA ${ }^{\mathrm{V}}$ and its oxygen-analogue $\mathrm{DMA}^{\mathrm{V}}$ were detected. Detailed speciation studies in the absence and the presence of differentiated Caco- 2 cells indicate a spontaneous, about $11 \%$, degradation of thio-DMA $\mathrm{V}^{\mathrm{V}}$ in the absence of cells and an about $20 \%$ metabolism of thio-DMA ${ }^{\mathrm{V}}$ by the cells. These speciation studies allow the conclusion that thio-DMA ${ }^{\mathrm{V}}$ itself is transported across the intestinal barrier, since on both sides of the barrier thio-DMA ${ }^{\mathrm{V}}$ was detected. Inside the cells several reactions can be considered. Thio$\mathrm{DMA}^{\mathrm{V}}$ might be metabolised to $\mathrm{DMA}^{\mathrm{V}}$ but also to trivalent intermediates, including $\mathrm{DMA}^{\mathrm{III}}$ or $\mathrm{DMAG}^{\mathrm{III}}$, which could then be oxidized to $\mathrm{DMA}^{\mathrm{V}}$ after their transport out of the cells. Further intracellular speciation studies could prove these assumptions.

\section{Conclusion}

The two arsenosugar metabolites thio-DMA ${ }^{\mathrm{V}}$ and thio-DMAE ${ }^{\mathrm{V}}$ showed intestinal bioavailability similar to that of arsenite, and about 10-fold higher than that reported for arsenosugars in the same in vitro model. ${ }^{29}$ Thus, a presystemic metabolism of arsenosugars most likely strongly impacts arsenic intestinal bioavailability after arsenosugar intake and should therefore be considered when assessing the risks to human health related to the consumption of arsenosugar-containing food.

\section{Abbreviations}

$\begin{array}{ll}\text { oxo-DMAA }^{\mathrm{V}} & \text { oxo-dimethylarsenoacetate } \\ \text { oxo-DMAE }^{\mathrm{V}} & \text { oxo-dimethylarsenoethanol }\end{array}$ 
thio-DMAA ${ }^{\mathrm{V}}$ thio-dimethylarsenoacetate

thio-DMAE $^{\mathrm{V}}$ thio-dimethylarsenoethanol

$\mathrm{DMA}^{\mathrm{V}} \quad$ dimethylarsinic acid

DMA $^{\text {III dimethylarsinous acid }}$

DMAG $^{\text {III dimethylarsenic glutathione }}$

iAs $^{\mathrm{V}}$

ICP-QMS

$\mathrm{MMA}^{\mathrm{V}}$

MRP

$P_{\mathrm{c}}$

TEER

thio-DMA ${ }^{\mathrm{V}}$

arsenate monomethylarsonic acid

permeability coefficient

thio-dimethylarsinic acid inductively coupled plasma mass spectrometry

multidrug resistance associated proteins

transepithelial electrical resistance

\section{Conflict of interest statement}

We declare that we have no conflict of interest.

\section{Acknowledgements}

This work was supported by the DFG grant number SCHW 903/ 4-1, the Austrian Science Fund (FWF), project number I550N17, and the NRW Graduate School of Chemistry (WWU Muenster, Germany).

\section{References}

1 EFSA, Opinion of the Panel on Contaminants in the Food Chain (CONTAM), Scientific Opinion on Arsenic in Food, EFSA J., 2009, 7, 1351, 1-198.

2 IARC, IARC Monographs on the Evaluation of the Carcinogenic Risks to Humans, suppl. 7, Overall Evaluations of Carcinogenicity: An Updating of IARC Monographs, Lyon, France, 1987, vol. 1-42.

3 IARC, A review of human carcinogens - Part C: Arsenic, metals, fibres and dusts, IARC Monographs, Lyon, France, 2012, vol. 100, pp. 196-211.

4 K. A. Francesconi and D. Kuehnelt, Environmental Chemistry of Arsenic, in Books in Soils, Plants, and the environment, ed. W. T. Frankenberger Jr., Marcel Dekker Inc., New York, 2002, pp. 51-94.

5 R. Raml, W. Goessler, P. Traar, T. Ochi and K. A. Francesconi, Chem. Res. Toxicol., 2005, 18, 1444-1450.

6 F. Challenger, Chem. Rev., 1945, 36, 315-361.

7 T. Hayakawa, Y. Kobayashi, X. Cui and S. Hirano, Arch. Toxicol., 2005, 79, 183-191.

8 T. Watanabe and S. Hirano, Arch. Toxicol., 2013, 87, 969-979.

9 JECFA, Seventy-second report of the Joint FAO/WHO Expert Committee on Food Additives, World Health Organization, Geneva, Switzerland, 2011.

10 K. Rehman and H. Naranmandura, Metallomics, 2012, 4, 881-892.

11 S. Nesnow, B. C. Roop, G. Lambert, M. Kadiiska, R. P. Mason, W. R. Cullen and M. J. Mass, Chem. Res. Toxicol., 2002, 15, 1627-1634.
12 F. Ebert, A. Weiss, M. Bultemeyer, I. Hamann, A. Hartwig and T. Schwerdtle, Mutat. Res., 2011, 715, 32-41.

13 T. Schwerdtle, I. Walter, I. Mackiw and A. Hartwig, Carcinogenesis, 2003, 24, 967-974.

14 A. D. Kligerman, C. L. Doerr, A. H. Tennant, K. HarringtonBrock, J. W. Allen, E. Winkfield, P. Poorman-Allen, B. Kundu, K. Funasaka, B. C. Roop, M. J. Mass and D. M. DeMarini, Environ. Mol. Mutagen., 2003, 42, 192-205.

15 J. Bornhorst, F. Ebert, H. Lohren, H. U. Humpf, U. Karst and T. Schwerdtle, Metallomics, 2012, 4, 297-306.

16 H. R. Hansen, M. Jaspars and J. Feldmann, Analyst, 2004, 129, 1058-1064.

17 H. R. Hansen, A. Raab, M. Jaspars, B. F. Milne and J. Feldmann, Chem. Res. Toxicol., 2004, 17, 1086-1091.

18 H. R. Hansen, A. Raab, K. A. Francesconi and I. Feldmann, Environ. Sci. Technol., 2003, 37, 845-851.

19 E. M. Kenyon, M. F. Hughes, B. M. Adair, J. H. Highfill, E. A. Crecelius, H. J. Clewell and J. W. Yager, Toxicol. Appl. Pharmacol., 2008, 232, 448-455.

20 R. Raml, A. Rumpler, W. Goessler, M. Vahter, L. Li, T. Ochi and K. A. Francesconi, Toxicol. Appl. Pharmacol., 2007, 222, 374-380.

21 K. A. Francesconi, R. Tanggaard, C. J. McKenzie and W. Goessler, Clin. Chem., 2002, 48, 92-101.

22 R. Raml, G. Raber, A. Rumpler, T. Bauernhofer, W. Goessler and K. A. Francesconi, Chem. Res. Toxicol., 2009, 22, 1534-1540.

23 A. H. Ackerman, P. A. Creed, A. N. Parks, M. W. Fricke, C. A. Schwegel, J. T. Creed, D. T. Heitkemper and N. P. Velal, Environ. Sci. Technol., 2005, 39, 5241-5246.

24 K. M. Kubachka, M. C. Kohan, K. Herbin-Davis, J. T. Creed and D. J. Thomas, Toxicol. Appl. Pharmacol., 2009, 239, 137-143.

25 S. D. Conklin, A. H. Ackerman, M. W. Fricke, P. A. Creed, J. T. Creed, M. C. Kohan, K. Herbin-Davis and D. J. Thomas, Analyst, 2006, 131, 648-655.

26 N. Bu, H. Y. Wang, W. H. Hao, X. Liu, S. Xu, B. Wu, Y. Anan, Y. Ogra, Y. J. Lou and H. Naranmandura, Metallomics, 2011, 3, 1064-1073.

27 T. S. Pinyayev, M. J. Kohan, K. Herbin-Davis, J. T. Creed and D. J. Thomas, Chem. Res. Toxicol., 2011, 24, 475-477.

28 T. Van de Wiele, C. M. Gallawa, K. M. Kubachka, J. T. Creed, N. Basta, E. A. Dayton, S. Whitacre, G. Du Laing and K. Bradham, Environ. Health Perspect., 2010, 118, 1004-1009.

29 L. Leffers, F. Ebert, M. S. Taleshi, K. A. Francesconi and T. Schwerdtle, Mol. Nutr. Food Res., 2013, DOI: 10.1002/ mnfr.201200821, Epub 8 Apr. 2013.

30 P. Shah, V. Jogani, T. Bagchi and A. Misra, Biotechnol. Prog., 2006, 22, 186-198.

31 P. Artursson and R. T. Borchardt, Pharm. Res., 1997, 14, 1655-1658.

32 E. Le Ferrec, C. Chesne, P. Artusson, D. Brayden, G. Fabre, P. Gires, F. Guillou, M. Rousset, W. Rubas and M. L. Scarino, Altern. Lab. Anim., 2001, 29, 649-668.

33 M. Calatayud, V. Devesa, R. Montoro and D. Velez, Toxicol. Lett., 2011, 204, 127-133.

34 M. Calatayud, M. Vazquez, V. Devesa and D. Velez, Chem. Res. Toxicol., 2012, 25, 2654-2662. 
35 J. M. Laparra, D. Velez, R. Barbera, L. Granero, A. Polache, R. Montoro and R. Farre, Toxicol. In Vitro, 2006, 20, 658-663.

36 M. Calatayud, J. Gimeno, D. Velez, V. Devesa and R. Montoro, Chem. Res. Toxicol., 2010, 23, 547-556.

37 M. Bartel, F. Ebert, L. Leffers, U. Karst and T. Schwerdtle, J. Toxicol., 2011, DOI: 10.1155/2011/373141, Epub 11 Oct. 2011.

38 K. A. Francesconi, J. S. Edmonds and R. V. Stick, Sci. Total Environ., 1989, 79, 59-67.

39 J. S. Edmonds, K. A. Francesconi and J. A. Hansen, Experientia, 1982, 38, 643-644.

40 J. Bornhorst, C. A. Wehe, S. Huwel, U. Karst, H. J. Galla and T. Schwerdtle, J. Biol. Chem., 2012, 287, 17140-17151.

41 Y. Sambuy, I. De Angelis, G. Ranaldi, M. L. Scarino, A. Stammati and F. Zucco, Cell Biol. Toxicol., 2005, 21, 1-26. 42 D. A. Volpe, J. Pharm. Sci., 2008, 97, 712-725.

43 P. Artursson and J. Karlsson, Biochem. Biophys. Res. Commun., 1991, 175, 880-885.

44 J. M. Laparra, D. Velez, R. Barbera, R. Montoro and R. Farre, Toxicol. In Vitro, 2005, 19, 1071-1078.
45 J. Laparra, D. Velez, R. Barbera, R. Montoro and R. Farre, J. Agric. Food Chem., 2007, 55, 5892-5897.

46 M. Calatayud, J. A. Barrios, D. Velez and V. Devesa, Chem. Res. Toxicol., 2012, 25, 446-453.

47 M. Calatayud, D. Velez and V. Devesa, Chem. Res. Toxicol., 2012, 25, 2402-2411.

48 H. Franke, H. J. Galla and C. T. Beuckmann, Brain Res., 1999, 818, 65-71.

49 P. Alava, F. Tack, G. D. Laing and T. Van de Wiele, J. Hazard. Mater., 2012, DOI: 10.1016/j.jhazmat.2012.05.042.

50 S. V. Kala, M. W. Neely, G. Kala, C. I. Prater, D. W. Atwood, J. S. Rice and M. W. Lieberman, J. Biol. Chem., 2000, 275, 33404-33408.

51 X. Cui, Y. Kobayashi, T. Hayakawa and S. Hirano, Toxicol. Sci., 2004, 82, 478-487.

52 H. Naranmandura, M. W. Carew, S. Xu, J. Lee, E. M. Leslie, M. Weinfeld and X. C. Le, Chem. Res. Toxicol., 2011, 24, 1586-1596.

53 P. Artursson, K. Palm and K. Luthman, Adv. Drug Delivery Rev., 2001, 46, 27-43. 